\title{
How to reduce clinical expenses
}

\author{
Jinguo Wang ${ }^{1, a}$ and Na Wang ${ }^{2, b^{\star}}$ \\ ${ }^{1}$ the First Hospital of Jilin University, Changchun 130021, China; \\ 2 the First Hospital of Jilin University, Changchun 130021, China \\ awangjinguolily@163.com, bwangna080613@163.com
}

Keywords: Fairness, rationality, expense.

\begin{abstract}
Unnecessary medical examinations increase health care costs, waste resources, as well as cause the patient's physical and mental injury, which may result in medical disputes. Unreasonable medical examinations has become a limiting factor of the sustainable development of modern hospital. Rationality of medical examinations is the important official measure for evaluation of medical institutions.
\end{abstract}

\section{Introduction}

Medical examination is an important part of modern medical service is indispensable. In this paper, the rationality of the medical examination and the related problems of research, can help people more in-depth and systematic understanding of the basic features of the medical check behavior and internal mechanism, benefit to guide and standardize the practice of medical examination, to improve medical service quality, reduce medical service cost and establish the harmonious doctor-patient relationship, can also be used for medical and health departments set up a scientific medical examination provides some references for the supervision and management of the specification.

\section{Text}

The development of modern medical service has, to a certain extent without the participation of medical examination behavior. Medical checks daily application, however, but there are many non-standard, unreasonable phenomenon, mainly show the people often say that "excessive examination", check "unnecessary" form, etc [1]. medical examination is not standard, unreasonable use, not only to improve the level of clinical diagnosis and treatment quality, waste the limited health care resources, and increase the patient's physical and mental pain and economic burden, large medical equipment inspection cost has become the second after drug prices artificially high lead to the factor of "expensive", could eventually lead to the development of medical examination technology into a "bottleneck".This makes people have to think about: what kind of medical examination is reasonable? In the face of medical check the rationality of the problem, for many years, many medical experts and scholars gave full attention to related disciplines and thinking, also made a tireless study and research.

At present, home and abroad about the medical examination of rationality, the main technology in disease diagnosis and treatment level focus on the proper use of the medical instrument and technology projects, namely how to rational use of medical inspection equipment and technology projects to achieve the best effect of diagnosis, and some of the specific disease how to diagnosis the rational use of medical instrument and so on. At the same time, some scholars also found that unreasonable check is not only a medical check technical problems, also can cause or aggravate certain physical and mental health damage, patients may also involve medical tort law and medical ethics issues, and therefore the cause of medical disputes or doctor-patient contradiction, become a key factor for the sustainable development of modern hospital [2]. Although scholars on the rationality of the medical examination made many fruitful research results, however, this paper argues that at present these results still has the following several aspects of the research is not enough, need further thinking and exploring. 
This thesis is based on and respect existing research results about the health check, do not go into specific medical check project technical attributes and use skills question discussion, not to demonstrate specific patients or specific health concerns how to apply the rationality of medical examination means for clinical diagnosis, mainly around the medical institutions in medical check may involve in the actual use of economic, social medicine or medical sociology such as ethical and legal problems accordingly. This article mainly points three levels gradually open issues related to research. First analyzes the basic connotation of the rationality of the rational and expounded the value rationality and instrumental rationality of medical examination, unlimited rationality and limited rationality and formal rationality and essential rationality, procedural rationality and rational concepts and forms as a result, after confirming the reasonableness of the hospital diagnosis and treatment

Rational orientation and the main factors influencing the medical check reasonable implementation, and then discusses the basic standard of medical check the rationality of evaluation. Rationality in the health check issues related to in-depth research, mainly includes the perspective of the tort liability act in our country medical examination of infringement and its liability, medical inspection technology of medical relations materialized and check the patients right of informed consent in special medical protection, as well as the clinical pathway implementation step by step in China under the new situation, the medical check construction problems such as the concept of marketing and subculture. Study as the breakthrough point, through some argument medical check rationality and legitimacy, ethical, and social relationship, thinking about how to standardize and guide the reasonable implementation of the strategy and method of the medical examination [3].

To the empirical analysis of the rationality of the medical examination, first using the individual interview method to survey both doctors to subjective value judgment on the rationality of medical examination, and the subjective evaluation of department of medical and job satisfaction. Then, collected from patients in the hospital medical information, to analyze the different characteristics of hospitalized patients by using different categories of medical examination and the corresponding form than the cost of the project level, medical check total cost accounting for the proportion of the total cost in hospital, health inspection fees and expenses for medicine, interpretation of the hospitalized patients for clinical inspection, radiation imaging major medical check the use effect of the project. Finally, based on the above empirical data, analyze the medical check medical examination in the practice rationality evaluation of multidimensionality, patients participate in medical check rationality evaluation of subjectivity, as well as the patients right of informed consent in the medical check process, interest is the highest authority, and discussed the medical check fee burden of clinical application problems and may cause adverse effects, thus for the relevant departments of medical examination behavior norms and guidance provides some practical references.

According to the literature content analysis of these have certain reference significance and learning related literature has carried on the arrangement and classification, through the literature read and digest, refining, sublimation, combined with modern medical examination professional characteristics, and demonstrates the rationality of medical examination and its related problems of understanding and views. In this paper for medical check rationality problem of fundamental and pilot study, to standardize and guide the medical behavior of medical institutions have the very strong practical significance and theory value.

Medical check rationality through interpretation of the rational connotation, which is beneficial to change the biased understanding of medical examination behavior, cultivation of medical check rationality more comprehensive and systematic cognitive concept, thus rationality for medical check other related research laid the logical starting point.

By analyzing the main factors influencing the medical examination and reasonable implementation, not only for medical institutions to introduce and develop new technology of medical examination project provides feasibility idea, also is helpful for medical personnel timely adjust medical check supply strategy and improve the pertinence and effectiveness of supply. 
By discussing the rationality of the medical examination evaluation standard, can not only provide the competent department of public health medical check regulation theory resources, also is advantageous to the medical institutions have a destination to guide the implementation of the medical examination behavior and the choice of appropriate medical examination technology, so as to reduce or avoid unreasonable medical examination, save the limited health resources, improve the comprehensive efficiency of medical resources.Based on the related research of medical check rationality, can deepen the cognition to the legitimacy and ethical medical examination, help to expand medical checks the idea of sustainable development, set up the good social image of medical institutions, to build a harmonious medical environment.

Through the empirical analysis on the rationality of medical examination, not only helps to deepen the understanding of medical check rationality of the related theoretical study and grasp, are a helpful aid in getting to know the actual use of the medical examination and found that there were problems and the insufficiency, at the same time, access to empirical data and analysis and source of topic selection of medical examination for further studies.

Instrumental rationality value behavior, means, method, technology, the process of empirical and quantitative, is advocating the best solutions and best means and the best efficiency objective rationality. Instrumental rationality have a very clear and precise meaning, represents the choose the right means to achieve your intended to achieve the purpose of, it has nothing to do with the purpose to choose, no matter what is the purpose. Correspond to instrumental rationality and value rationality certainly man is the only main body of the world, but also perform any ACTS against the subject of object, subject and object of blending and mixing. Rational value certainly means the utilitarian purpose, but beyond the utilitarian, emphasize the justness and means of legitimacy of motivation, emphasize people ultimate concern should be to object, think that everything should be in people's behavior in order to maintain, development and realize the interests of the people's economic, political and cultural harmony, is to protect human dignity, enhance the value of people, showing people the meaning of existence, is to promote better survival, development and improvement. Visible, the behavior under rational value is in accordance with the requirements of some kind of belief, faith, or ideal and engaged in the behavior of required to realize this belief or also must be in conformity with the value of the ideal means. Tool rationality and value rationality on the reality state has a certain difference, but the state ought to be, as both the two aspects of awareness, which contain rational thinking and rational human activity, and mutual penetration, interweave each other, interdependent and harmonious unity. The existence of instrumental rationality, realized the phases of their own living conditions improving, this not only to achieve the sublimation of the value rationality to provide real support, has prompted new rational value goal and establish. At the same time, instrumental rationality embodies the subject of object and manage regularity of cognition, thus gradually formed the basis of science, technology, science, applied science, etc., constitute the basis of the accumulation and development of human civilization, and thus was born, refining and produce new value rationality.

Therefore, a certain sense, the distinction between instrumental rationality and value rationality is only exists in the concept of theoretical derivation and logical deduction.In human's rational practice, rational value if not on the basis of instrumental rationality, it may be false and the rationality of vacant, is difficult to achieve [4]. Also, there is no rational value guide and standardize the tools is rational ignorance and blind, can't get the sustainable and healthy development. However, when people are the pursuit of efficiency and the process of implementation of the technology control, instrumental rationality to greatly expand gradually, instrumental rationality by means of liberation of humanity is increasingly degraded to dominate, control force and chains, instrumental rationality presented the trend of increasingly replaced by technical rationality or scientific rationality, and thereby's humanistic attribute value rationality contradiction happened. The internal rational contradiction in the field of different breed different manifestations of conflicts and disputes, has become one of the root causes of crisis in modern society.

Formal rationality and essential rationality is weber, two very important concepts in theory of the whole society, at the same time, and as a major form of thinking, to analyze the problem in economic 
sociology, sociology of law sociology and administrative branch discipline of sociology of by fully using the different level.Formal rationality and essential rationality has several fundamental difference, but there are also many inevitable connection.Form the rational demand behavior standardization, the standard is the basis of decision-making, emphasizing on the unity of the relatively fixed standard to evaluate rationality of different action or behavior results, and relative lack of flexibility and mobility; In contrast with the specific essential rational analysis, to meet the practical and pragmatic as a basis for the decision-making, emphasize the pragmatic of behavior, that behavior should comply with the particular area, certain people and certain cultural specific scenarios such as people's behavior habits and values, which according to the different situation to make analysis and evaluation on the rationality of different processing behavior and behavior, thus lack of relatively uniform standard and quantitative standard.In fact, the formal rationality and essential rationality have neither, or there are no absolute separation, each person's behavior in the logic or the specific types of behavior, all at the same time there is the concept of formal rationality and essential rationality and thinking, and influence each other, and dependent on each other.Only try to conform to the actual and humane norms of behavior that can be accepted by people, also can be effective, at the same time, to standardize the behaviour can be corrected, education, guidance, and evaluation of human behavior, is advantageous to the absorption of discontent and fairness, to effectively implement and reflect the real reason.

\section{Summary}

Medical examination technology due to the development of science and technology, also with the development of modern science and technology and some automation, intelligent and systematic upgrading, as a result, the rationality of the medical examination evaluation should have the view of history, on the basis of medical material and technical conditions at that time as well as the specific historical conditions of people's value concept and the influence factors such as health concept to judge, that is to say, the rationality of the medical examination evaluation criterion should be history, change, not eternal, immutable.Don't be isolated from the big social system to assess the rationality of medical examination.Medical service system is the subsystem of the society, the reasonable implementation of the medical examination from many social factors, the influence of different areas of the supply of medical examination service levels, types and mode have corresponding difference, specific people medical examination service demand and utility standards tend to also have much difference.If leave medical check the implementation of specific area reality, will lose the rationality of the medical examination evaluation scientific and objective.

\section{References}

[1] Lujun Gao, Ling Liu, Jihui Zhang, Lidan Hou. Building of Smart Home Medical System Based on Internet of Things [A]. Proceedings of 2016 Cloud Computing and Internet of Things Conference (CCIOTC2016) [C]. 2016

[2] Xianxiang Chen, Zhen Fang, Ren Ren, Shanhong Xia, Yangmin Qian, Huaiyong Li, Lili Tian, Wenxi Zhai, Weibing Xu, Hui Gao. Simple and efficient baseline removal method for a smartphone based ECG detection device [A]. Proceedings of 2013 International Conference on Future Computer and Information Technology (ICFCIT 2013) [C]. 2013

[3] Fang Qingwei. Research on Evaluation Index System of Management Effectiveness on Hospital Human Resource Based on Balanced Scorecard [A]. Proceedings of 2011 International Conference on Fuzzy Systems and Neural Computing (FSNC 2011 V2) [C]. 2011 
[4] Thepparit Sinthamrongruk, Napat Harnpomchai. Determination of optimal number of doctors and appointment interval for an appointment [A]. Proceedings of the International Conference on Information Engineering and Education Science [C]. 2014 